\title{
Administration of epidural labor analgesia is not associated with a decreased risk of postpartum depression in an urban Canadian population of mothers: a secondary analysis of prospective cohort data
}

This article was published in the following Dove Press journal: Local and Regional Anesthesia

3I October 2017

Number of times this article has been viewed

\author{
Marissa Nahirney' \\ Amy Metcalfe ${ }^{2}$ \\ Katie H Chaput ${ }^{3}$ \\ 'O'Brien Centre, ${ }^{2} \mathrm{Obstetrics}$ and \\ Gynecology, ${ }^{3}$ Alberta Children's \\ Hospital Research Institute and \\ Pediatrics, Cumming School of \\ Medicine, University of Calgary, \\ Calgary, AB, Canada
}

Correspondence: Katie H Chaput Alberta Children's Hospital Research Institute and Pediatrics, Cumming School of Medicine, University of Calgary, HMRB 2, 3330 Hospital Drive NW, Calgary, AB T2J 3B4, Canada

Tel + I 4039980458

Email khchaput@ucalgary.ca
Background: Postpartum depression (PPD) is a common complication of pregnancy, affecting approximately $13 \%$ of mothers internationally. Previous research has examined whether epidural analgesia used for pain control during labor and birth is associated with a lower risk of PPD, but reports conflicting results and may have suffered from methodological shortcomings. Our study aimed to prospectively assess whether epidural analgesia is associated with a lower risk of PPD (at either 6 weeks or 6 months postpartum) after attempting to adequately adjust for selection bias and confounding variables.

Methods: We conducted a secondary analysis of a prospective cohort of urban Canadian mothers who were recruited at birth in Calgary, Canada, in 2010, for a primary study on predictors of PPD. Mothers with full-term, singleton infants who did not require neonatal intensive care unit admission of $>24$ hours were included, and filled out questionnaires at birth, 6 weeks and 6 months postpartum including demographics, birth data, maternal and infant physical health, lifestyle, breastfeeding and maternal mental health. Descriptive statistics were calculated for participant characteristics and to identify potential confounder variables. Multivariable logistic regression analysis was conducted to assess whether epidural analgesia is associated with PPD after controlling for available confounding variables.

Results: Our study included 206 mothers who had vaginal deliveries and were free of depression at delivery. We found an incidence of PPD of $13.3 \%(n=27)$ and no statistically significant association between epidural use and PPD, regardless of adjustment for potential confounding variables (unadjusted odds ratio [OR] 0.86, 95\% confidence interval [CI] 0.69-1.22; adjusted OR (for body mass index 1.04, 95\% CI 0.40-2.77).

Conclusion: We did not observe a significant association between epidural use and PPD. While the CIs are wide, we do not believe that this masks a clinically relevant association, and as such, the risks and benefits of epidural analgesia communicated to women during labor and delivery should not be modified.

Keywords: epidural analgesia, labor analgesia, postpartum depression, maternal mental health

\section{Introduction}

Postpartum depression (PPD) is a common and debilitating illness affecting $15 \%$ of women in the first 6-12 months postpartum that can impact daily functioning. ${ }^{1-3}$ Women who have experienced marital difficulties, stressful life events and antenatal depression, 
along with those from lower socioeconomic brackets, those with higher than normal body mass index (BMI) and/or a history of abuse are at greater risk for PPD. ${ }^{1}$ Impacts of PPD extend beyond the mother to long-term cognitive, social and motor development of the child. ${ }^{3}$ Women suffering from PPD are at greater risk of early breastfeeding cessation and strained mother-child relationships leading to the development of problematic child behavior. ${ }^{3-6}$ The etiology of PPD is complex, and previous research has implicated the pain and stress of labor and delivery as a potential contributing factor. ${ }^{7}$ Eisenach et $\mathrm{al}^{9}$ reported a heightened risk of PPD if a woman's acute pain was more severe. In addition, postpartum pain may be a contributing factor to PPD; roughly $11 \%$ of women report the onset of severe acute pain after delivery, which is associated with depressive symptomology. ${ }^{9,10}$

Epidural analgesia is widely employed as a safe and effective method of pain management during parturition, but it is not without risks. ${ }^{8,11}$ Epidural analgesia use has been associated with longer labor, infection, the need for instrumental delivery, higher risk of labor complications, acute hypotension, headaches, dural puncture complications and, very rarely, paralysis. ${ }^{12-14}$ However, it remains one of the most broadly used pain control methods for childbirth and is highly effective. ${ }^{10}$ It has been suggested that adequate pain control during labor and birth might decrease the risk of PPD, via lessening the traumatic experience of birth. ${ }^{7}$ Three recent studies examined the association between epidural analgesia and PPD, with inconsistent results. ${ }^{15-17}$ A Danish study of 185 women found that epidural analgesia was associated with lower odds of PPD immediately after delivery (odds ratio [OR] 0.25, 95\% confidence interval [CI] 0.09-0.71); however, the protective association was not sustained beyond 4 weeks postpartum (OR 0.74, 95\% CI 0.18-3.00). ${ }^{17} \mathrm{~A}$ larger case-control study in a sample of 479 women from Singapore showed similar short-term results, indicating lower odds of PPD at 4-8 weeks postpartum among those who received epidural labor analgesia, controlling for history of depression and other PPD risk factors (OR 0.47, $P=0.0078) .{ }^{15}$ The retrospective nature of the study prevents the assessment of whether epidural is protective against PPD, or if women with depression are simply more likely to request epidural analgesia. In a longer, prospective study of 214 women delivering babies at a Beijing hospital, Ding et al reported significantly lower odds of PPD among women who received epidural labor analgesia (OR $0.31,95 \%$ CI 0.12-0.82). ${ }^{16}$ However, the study may have been subject to selection bias, due to systematic exclusion of obese patients and differential loss to follow-up that favored retention of the nondepressed mothers in the epidural group. ${ }^{18}$ Furthermore, the study may have lacked appropriate control for measured confounding variables, which likely biased the reported result. ${ }^{18}$

Our study aimed to assess whether epidural analgesia is associated with a lower risk of PPD at 6 months postpartum, among a group of mothers with no depression at delivery, after control for selection bias and appropriate adjustment for confounding. Determining whether epidural analgesia is associated with reduced odds of PPD is essential to the appropriate communication about the risks and benefits of epidural use to women prior to and during labor.

\section{Subjects and methods}

This study was a secondary analysis of data from the Happy Baby Cohort - a prospective community-based cohort of women delivering healthy, full-term infants in one of the three active maternity hospitals (Foothills Medical Centre, Peter Lougheed Centre, and Rockyview General Hospital) in Calgary, Alberta, Canada in 2010; detailed methods of which are published elsewhere. ${ }^{19}$ Calgary is a large urban center with a 2010 population of approximately 1.1 million. The study was approved by the Conjoint Health Research Ethics Board at the University of Calgary. Women were approached about the study on admission to the postpartum units, and 979 mothers indicated interest in hearing about the study from researchers. A sample of 446 mothers provided written informed consent, and they were enrolled in the original cohort study, in which participants completed paper and/ or electronic questionnaires at birth, 6 weeks and 6 months postpartum. ${ }^{19}$ Of the 446 mothers in the cohort, 113 were excluded from the current study due to cesarean deliveries as epidurals are not standard forms for pain control in this population, 41 were excluded because they scored at least 13 on the Edinburgh Postnatal Depression Scale (EPDS) at delivery, indicating a strong possibility of preexisting depression prior to epidural administration. A score of 13 or greater on the EPDS at delivery is a validated measure of depression in antenatal populations and was used to ensure a depressionfree sample at the study onset. ${ }^{16}$ A further 86 participants withdrew from the study or were lost to follow up over the 6-month follow-up period. A final convenience sample of 206 participants was included in the current analysis.

\section{Variables and definitions}

The study exposure was the use of epidural analgesia in labor and delivery and was measured dichotomously (yes/ no). Our primary outcome was PPD, defined as a score of 10 or greater on the EPDS at either 6 weeks or 6 months. ${ }^{20}$ Although not a diagnostic tool, the EPDS has been validated and deemed reliable as a screen for PPD in Alberta 
and other Canadian provinces. ${ }^{21,22}$ It differentiates between non-pathological postpartum "blues" symptoms and those of PPD, more effectively than other depression measures. ${ }^{22}$ Other maternal characteristics were measured as potential confounding variables, including age, parity, socioeconomic status (marital status, education, annual household income, language spoken at home, home ownership), other pain control modalities, satisfaction with birth experience and pre-pregnancy BMI, measured at 6 weeks postpartum. The 6-week postpartum measure of BMI was used because it was the most complete measure of BMI available for the sample, and it likely reflects a combination of pre-pregnancy BMI, gestational weight gain and general health status in the postpartum period. As there are currently no standardized categories for BMI among postpartum and breastfeeding women, BMI was summarized categorically, but included in analyses as a continuous variable whenever possible.

\section{Statistical analysis}

Descriptive statistics were expressed as means for continuous variables and proportions for categorical and dichotomous variables. All missing data were handled through case-wise deletion. Multivariable logistic regression analysis was conducted using a backward elimination method, wherein the initial model includes the maximum number of potential confounding variables and the model is reduced sequentially until only significant variables remain. ${ }^{17,23}$ Variables were included in the model as possible confounding variables if they were associated with a difference of $2 \%$ or greater in the proportion of both epidural use and PPD. All statistical analyses were conducted using STATA-IC version 13.1 (StataCorp LP, College Station, TX, USA), at an alpha level of 5\%. Given our sample size of 206 women, we had adequate power $(>80 \%)$ to detect a $30 \%$ difference in our primary outcome.

\section{Results}

Characteristics of the cohort were representative of an Albertan and Canadian maternal population in previous publications ${ }^{18}$ (Figure 1). The majority of participants were young mothers, having delivered between the ages of 18 and 35 years $(79.3 \%)$. A large proportion of the sample was married, financially stable, owned their homes and had lived in Canada for at least 5 years, consistent with the maternal population in Alberta (Table 1). The mean BMI reported at 6 weeks postpartum was $26.1 \mathrm{~kg} / \mathrm{m}^{2}\left(\mathrm{SD} 49 \mathrm{~kg} / \mathrm{m}^{2}\right)$. Maternal body weight was missing for $18 \%$ of the participants. Approximately half of those who reported their body weight had BMIs of $25 \mathrm{~kg} / \mathrm{m}^{2}$ or higher (42.6\%), as expected for the early postpartum period.

The overall incidence of epidural use was $57.5 \%(\mathrm{n}=118)$. Proportions of epidural use differing by at least $2 \%$ from the overall proportion were observed among mothers with lower annual household income, those who did not own their homes, spoke a language other than English at home, had higher BMI and were primiparous, whereas Epidural analgesia use did not vary by more than $2 \%$ across maternal education and maternal age (Table 2).

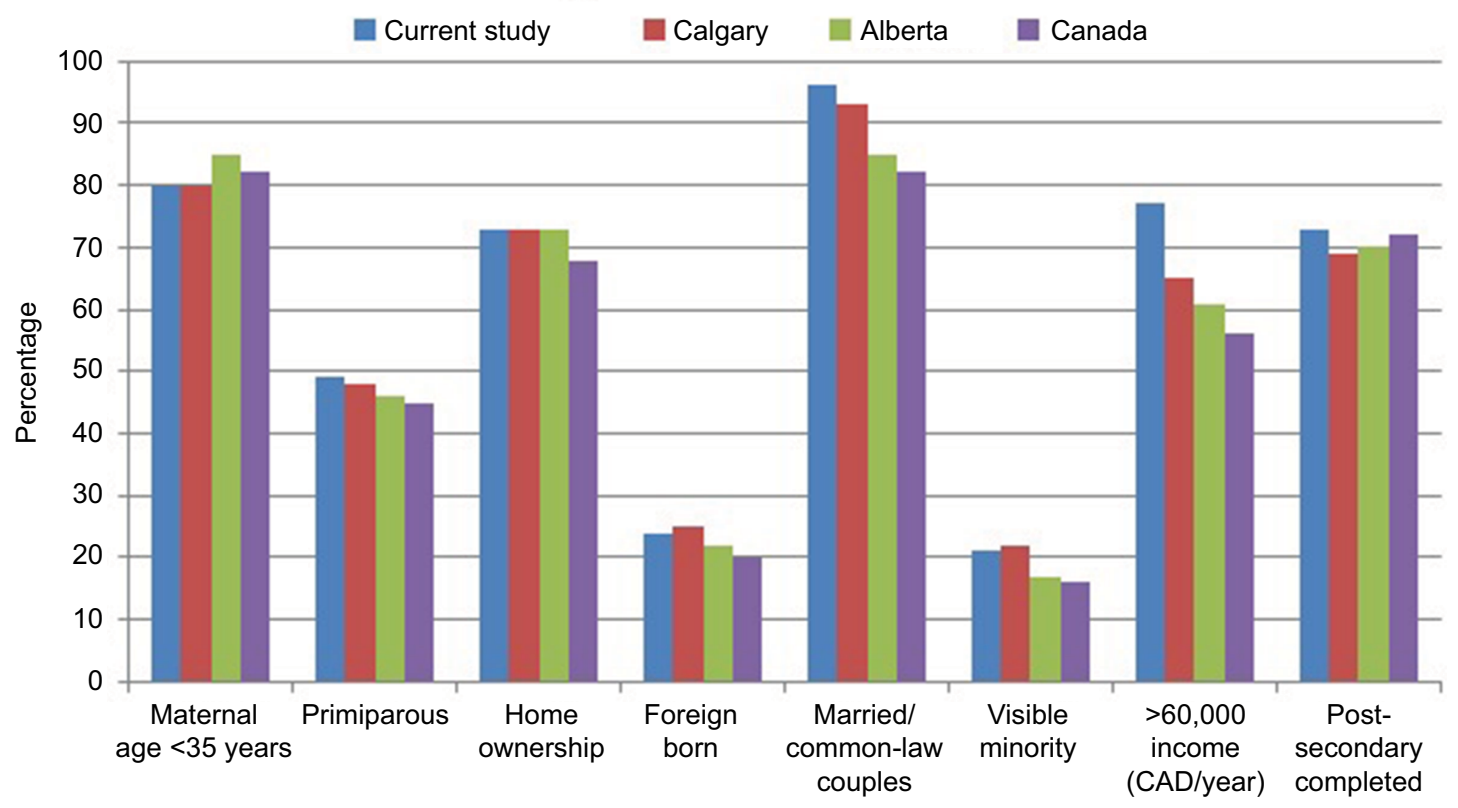

Participant characteristics

Figure I Comparison of participant characteristics to population values. 
The incidence of EPDS scores of 10 or greater was $12.9 \%$ at 6 weeks and, $13.3 \%(n=27)$ of women screened positive for PPD at either 6 weeks or 6 months postpartum, with slightly higher proportions of PPD among older mothers $(16.7 \%, 95 \%$ CI $8.0-31.3 \%)$ and those with higher BMI $(18.6 \%, 95 \%$ CI 11.3-29.3\%). Maternal age, parity, income and BMI were determined to be potential confounding variables as they demonstrated some association with both epidural use and PPD and were not intermediate variables. BMI was included in the regression modeling analysis as a continuous variable,

Table I Participant characteristics

\begin{tabular}{lll}
\hline Variable & Category & Percentage \\
\hline Maternal education & High school or less & 16.3 \\
& At least some postsecondary & 83.6 \\
Marital status & Single & 2.4 \\
& Married/common law & 97.6 \\
Household income & Less than \$60k annually & 23.7 \\
(CAD) & \$60k or more annually & 76.2 \\
Home ownership & Owns home & 71.1 \\
& Does not own home & 28.9 \\
Primary language & English & 88.0 \\
spoken at home & Others & 12.0 \\
Living in Canada & Less than 5 years & 5.2 \\
& More than 5 years & 94.8 \\
Parity & Primiparous & 52.3 \\
& Multiparous & 47.7 \\
Maternal age (years) & 35 and over & 20.6 \\
& I8-<35 & 79.3 \\
Body mass index & Mean = 30.I, SD (4.9) \\
$\left(\mathrm{kg} / \mathrm{m}^{2}\right)(\mathrm{n}=169)^{*}$ & 25 and under & 57.4 \\
& Over 25 & 42.6 \\
\hline Mon & Mean 24.4, SD (4.2)
\end{tabular}

Note: ${ }^{*} \mathrm{~N}=206$ unless otherwise indicated.

Abbreviation: SD, standard deviation. as there are no standardized categories for classification of maternal BMI in the postpartum. Multivariable logistic regression (Table 3 ) revealed that parity and annual household income were not independently associated with PPD in the model, and as such, they were not retained in the final model. BMI was the only potential confounder retained in the adjusted model. Controlling for BMI, epidural use was not significantly associated with PPD (OR 1.04, 95\% CI 0.39-2.77). BMI, however, remained a significant predictor of PPD, changing the odds of PPD by a factor of 1.13 for every point of BMI increase (OR 1.13, 95\% CI 1.03-1.25, $P=0.01$ ).

\section{Discussion}

Our study supports the proposed hypothesis that there is no significant association between epidural analgesia and PPD provided that selection biases are minimized by appropriate inclusions and measured confounding is adequately controlled.

The current study aimed to reassess the conflicting results obtained in two previous studies, in a Canadian population, using a prospective cohort of mothers who were free of depression at delivery, and with a definition of depression that spanned the most crucial 6 postpartum months. Hiltunen et al measured PPD using the EPDS at 3 days and 6 weeks

Table 3 Multivariable logistic regression models

\begin{tabular}{lllll}
\hline Outcome: PPD & OR & P-value & \multicolumn{2}{l}{$95 \%$ Cl } \\
\hline Epidural & 0.82 (crude) & 0.213 & 0.69 & 1.22 \\
Epidural & 1.04 (adjusted) & 0.946 & 0.40 & 2.77 \\
BMI (continuous) & 1.13 & 0.013 & 1.02 & 1.25 \\
\hline
\end{tabular}

Abbreviations: $\mathrm{BMI}$, body mass index; $\mathrm{Cl}$, confidence interval; $\mathrm{OR}$, odds ratio; PPD, postpartum depression.

Table 2 Associations of epidural analgesia and PPD with potential confounding variables

\begin{tabular}{|c|c|c|c|}
\hline Variables & Categories & $\begin{array}{l}\text { Percentage having } \\
\text { received epidural }\end{array}$ & $\begin{array}{l}\text { Percentage with PPD } \\
(E P D S \geq 10)\end{array}$ \\
\hline Overall & & 57.5 & 13.3 \\
\hline \multirow[t]{2}{*}{ Maternal education } & High school or less & 57.9 & 16.0 \\
\hline & Some postsecondary & 57.4 & 12.9 \\
\hline \multirow[t]{2}{*}{ Maternal age } & $18-<35$ years & 58.9 & 12.4 \\
\hline & 35 and over & 52.1 & 16.7 \\
\hline \multirow[t]{2}{*}{ Annual household income (CAD) } & Less than $\$ 60 \mathrm{k}$ & 62.2 & 13.3 \\
\hline & $\$ 60 \mathrm{k}$ or more & 55.9 & II.I \\
\hline \multirow[t]{2}{*}{ Language spoken at home } & English & 58.5 & 13.0 \\
\hline & Others & 50.0 & 13.3 \\
\hline \multirow[t]{2}{*}{ Home ownership } & Owns home & 53.3 & $1 \mathrm{I} .4$ \\
\hline & Does not own home & 67.2 & 13.3 \\
\hline \multirow[t]{2}{*}{ Parity } & Primiparous & 67.2 & 11.5 \\
\hline & Multiparous & 46.8 & 15.4 \\
\hline \multirow{2}{*}{ Body mass index $\left(\mathrm{kg} / \mathrm{m}^{2}\right)(\mathrm{n}=169)$} & 25 and under & 38.5 & 7.1 \\
\hline & Over 25 & 61.5 & 18.6 \\
\hline
\end{tabular}

Abbreviations: EPDS, Edinburgh Postnatal Depression Scale; PPD, postpartum depression. 
postpartum, while Sng et $\mathrm{al}^{15}$ measured depressive symptoms at 4-8 weeks. ${ }^{17}$ Their findings of a protective association of epidural analgesia prior to, but not beyond 8 weeks postpartum, are consistent with our results. Our study screened for PPD three times throughout the course of the study; this is important to capture the true incidence of this condition, as many women develop PPD after the first 6 weeks postpartum and experience "baby blues" rather than true PPD in the first 3 weeks after delivery. We measured PPD incidence in our sample at $13.3 \%$, similar to the Hiltunen et al's study (13.0\%), and consistent with other international findings. ${ }^{1,17}$ We are confident that our screening methods classified PPD accurately. Compared with previous studies, our sample had similar use of epidural labor analgesia $(57.5 \%)$, between the roughly $50 \%$ incidence of epidural analgesia use in two previous studies and lower than one previous study $(67.8 \%) .{ }^{15-17}$ Our findings support those of Hiltunen et al and confirm that there is no observed association between epidural labor analgesia use and PPD risk.

\section{Strengths and limitations}

The primary limitation of this study is that it was a secondary analysis requiring a convenience sample. Our sample size has limited the precision of our findings. The original cohort study from which ours was derived was designed to detect PPD as the primary outcome, and as such, the measurements used are valid for this analysis. Our sample was also restricted to mothers of infants who did not require neonatal intensive care unit admission. Mothers of infants in the neonatal intensive care unit are known to have a higher risk of PPD, and our findings cannot be extended to this population. Our sample size of 206 was similar to those of other studies designed to answer the same primary question, which were 214 and 185 participants, respectively, and our incidence of PPD was similar to the smaller previous study. ${ }^{16,17}$ Our study had adequate power (83.8\%) to detect a $30 \%$ difference in the outcome, similar to that reported in the study by Ding et al. ${ }^{16}$ Due to our sample size (and the subsequently wide CIs observed in our models), we cannot rule out the possibility that our models may be overfit. The small number of women experiencing PPD in this study also impeded our ability to adequately control for all potential confounding variables. While we do not believe that there is an association between epidural analgesia and PPD, we acknowledge that further, larger prospective studies on this topic are still needed to deduce the true association between these variables. A second limitation of the current study is the moderate level of missing BMI data. However, unlike the previous study in which all women with higher BMI were excluded from the study, all the mothers in our study, whether they reported BMI or not, were included in the analysis and contributed information to the final result. Our findings are thus not susceptible to the same selection biases that might have affected the study by Ding et al. Due to missing BMI data, complete control for confounding by BMI cannot be guaranteed. However, given that less than $20 \%$ of the BMI data were missing, we estimate that the likelihood of our result being affected by the missing data is small. An additional limitation of this and other studies of its kind is the lack of diagnostic information on PPD. A formal diagnosis of PPD was not feasible for a large cohort, and thus we are only able to present the associations between PPD screening results and the exposure. Future studies with diagnostic interviews would provide a more accurate measure of the association examined.

The strengths of our study include that it was prospective and that the sample of mothers were free of depression at delivery, allowing us to ensure that our exposure of epidural analgesia preceded the outcome of PPD. The use of the EPDS for the measurement of PPD is also valid and reliable in Canadian maternal populations, of which our cohort has been deemed representative. ${ }^{19}$ In addition, we were able to retain $80.7 \%$ of the sample in the study until 6 months postpartum.

\section{Conclusion}

Our study results counter the results of Ding et al, but echo the results of Hiltunen et al, that there is not an observed association between epidural analgesia and PPD. ${ }^{16,17}$ Further adequately powered studies should be conducted to further investigate this association with adjustment for additional confounding variables. At this point in time, there is insufficient evidence to change the information about the risks and benefits of epidural analgesia that are communicated to mothers prior to and during labor, who are considering epidural use. Epidural analgesia remains a safe and effective method of pain control during labor and birth. The significant association between BMI at 6 weeks postpartum and PPD should be investigated further as a primary objective in future research.

\section{Acknowledgment}

Funding for this study was awarded by the Women and Children's Health Research Institute, University of Alberta.

\section{Author contributions}

Marissa Nahirney contributed substantially to the project design, assisted with the analysis and was the primary author 
of the manuscript. Amy Metcalfe co-supervised the student project, contributed substantially to the design of the project and provided academic feedback on all manuscript drafts. Katie H Chaput supervised the development and write-up of the article, conducted the analysis and provided academic feedback to all manuscript drafts. Amy Metcalfe and Katie $\mathrm{H}$ Chaput attest to the integrity of the original data and the analysis reported in this manuscript. All authors contributed toward data analysis, drafting and critically revising the paper, gave final approval of the version to be published, and agree to be accountable for all aspects of the work.

\section{Disclosure}

The authors report no conflicts of interest in this work.

\section{References}

1. O'Hara M, Swain A. Rates and risk of post partum depression - a meta-analysis. Intern Rev psychiatr. 1996;8:37-54.

2. O'Hara WM. Postpartum depression: what we know. J Clin Psychol. 2009;65(12):1258-1269.

3. Lewis G. DSM-IV. Diagnostic and Statistical Manual of Mental Disorders. 4th ed. Washington, DC: American Psychiatric Association; 1996.

4. Grace SL, Evindar A, Stewart DE. The effect of postpartum depression on child cognitive development and behaviour: a review and critical analysis of the literature. Arch Womens Ment Health. 2003;6: 263-274.

5. Dennis C-L, McQueen K. The relationship between infant-feeding outcomes and postpartum depression: a qualitative systematic review. Pediatrics. 2009;123(4):e736-e751.

6. Davalos D, Yodn C, Tregellas H. Untreated prenatal maternal depression and the potential risks to offspring: a review. Arch Womens Ment Health. 2012;15(1):1-14.

7. Wisner KL. Double duty. Anesth Analg. 2014;119(2):219-221.

8. Lain S, Ford J, Hadfield R, Blyth F, Giles W, Roberts C. Trends in the use of Epidural analgesia in Australia. Obstet Anesth. 2009;29(3):148.
9. Eisenach J, Pan P, Smiley R, Lavand'homme P, Landau R, Houle T. Severity of acute pain after childbirth, but not type of delivery, predicts persistent pain and postpartum depression. Pain. 2008;140(1):87-94.

10. Korff M, Simon G. The relationship between pain and depression. $\mathrm{Br}$ J Psychiatry. 1996;168(30):101-108.

11. Hughes D, Simmons S, Brown J, Cyna A. Combined spinal-epidural versus epidural analgesia in labour. Birth. 2004;31(1):71.

12. Zhang J, Yancey MK, Klebanoff MA, Schwarz J, Schweitzer D. Does epidural analgesia prolong labor and increase risk of cesarean delivery? A natural experiment. Am J Obstet Gynecol. 2001;185(1):128-134.

13. Norris M, Grieco W, Borkowski M, Leighton B. Complications of labor analgesia. Anesth Analg. 1994;79(3):529-537.

14. Thorp J, Breedlove G. Epidural analgesia in labor: an evaluation of risks and benefits. Birth. 1996;23(2):63-83.

15. McGrady E, Litchfield K. Epidural analgesia in labour. Contin Educ Anesth Crit Care Pain. 2004;4(4):114-117.

16. Ding T, Wang D, Qu Y, Chen Q, Zhu S. Epidural labor analgesia is associated with a decreased risk of postpartum depression: a prospective cohort study. Anesth Analg. 2014;119(2):383-392.

17. Hiltunen P, Raudaskoski T, Ebeling H, Moilanen I. Does pain relief during delivery decrease the risk of postnatal depression? Acta Obstet Gynecol Scand. 2004;83(3):257-261.

18. Chaput K, Vinturache A. Methodologic concerns regarding a study concluding that epidural labor analgesia is associated with a decreased risk of postpartum depression. Anesth Analg. 2015;121(6):1682-1683.

19. Chaput KH, Nettel-Aguirre A, Mosto RC, Adair CE, Tough SC. Breastfeeding difficulties and supports and risk of postpartum depression in a cohort of women who have given birth in Calgary: a prospective cohort study. CMAJ Open. 2016;4(1):E103-E109.

20. Cox JL, Holden J, Sagovsky R. Detection of postnatal depression: development of the 10 item Edinburgh Postnatal Depression Scale. $\mathrm{Br}$ J Psychiatry. 1987;150:782-786.

21. Matthey S, Henshaw C, Elliott B. Variability in the use of cut-off scores and formats on the Edinburgh Postnatal Depression Scale: implications for clinical and research practice. Arch Womens Ment Health. 2006;9(6):309-315.

22. Jardri R, Pelta J, Maron M, et al. Predictive validation study of the Edinburgh Postnatal Depression Scale in the first week after delivery and risk analysis for postnatal depression. JAffect Disord. 2006;93(1-3):169-173.

23. Sun GW, Shook TL, Kay GL. Inappropriate use of bivariable analysis to screen risk factors for use in multivariable analysis. J Clin Epidemiol. 1996;49(8):907-916.
Local and Regional Anesthesia

Publish your work in this journal

Local and Regional Anesthesia is an international, peer-reviewed, open access journal publishing on the development, pharmacology, delivery and targeting and clinical use of local and regional anesthetics and analgesics. The journal is included in PubMed, and welcomes submitted papers covering original research, basic science, clinical studies,

\section{Dovepress}

reviews and evaluations, guidelines, expert opinion and commentary, case reports and extended reports. The manuscript management system is completely online and includes a very quick and fair peer-review system, which is all easy to use. Visit http://www.dovepress.com/ testimonials.php to read real quotes from published authors. 